\title{
ADAPTIVE ULTRASONIC SPECKLE REDUCTION BASED ON THE SLOPE-FACET MODEL
}

\author{
Huan-Chao Huang, ${ }^{*}$ Jau-Yuen Chen, ${ }^{\dagger}$ Sheng-De Wang* and Chung-Ming Chen ${ }^{\dagger}$ \\ *Institute of Electrical Engineering, National Taiwan University, Taipei, Taiwan; 'EPSON Palo Alto Laboratory, \\ Palo Alto, CA, USA; and Institute of Biomedical Engineering, National Taiwan University, Taipei, Taiwan
}

(Received 8 April 2002; revised 27 March 2003; in final form 31 March 2003)

\begin{abstract}
The flat-facet model has been implicitly assumed for the structure of the image surface by most conventional speckle-reduction algorithms. However, this model is rarely found in a real ultrasound (US) image. To preserve the higher order structures and to capture the spatially variant property of the speckle, a new adaptive speckle-reduction algorithm, called the symmetrical speckle-reduction filter (SSRF), was developed based on the slope-facet model. The basic idea of the SSRF was to estimate the uncorrupted signal on the largest symmetrical slope facet centered at each target pixel. The symmetry constraint ensured the correctness of the mean value. An empirical speckle model was incorporated to account for the nature of the speckle in US image. A two-stage despeckling strategy was employed to enhance the statistical reliability of each estimate by forming a union of a set of symmetrical despeckling windows. The proposed SSRF algorithm was compared with two filtered-based and one wavelet-based approaches and the experimental results showed that the proposed SSRF outperformed these three previous approaches in both the synthetic images and the clinical US images tested in this study. (ming@lotus.mc.ntu.edu.tw) (c) 2003 World Federation for Ultrasound in Medicine \& Biology.
\end{abstract}

Key Words: Ultrasound image, Speckle reduction, Flat-facet model, Slope-facet model, Symmetrical window, Signal-dependent noise, Speckle model.

\section{INTRODUCTION}

Ultrasonic speckle is a granular pattern caused by the constructive and destructive coherent interference of backscattered echoes from the scatterers smaller than the resolution size. Because of the tissue-dependent nature and the random mottle appearance, the speckle plays dual roles in the ultrasound (US) image analysis. For some clinical applications, the speckle serves as a crucial indicator for the diagnosis of diseases. For example, a liver with cirrhosis usually contains a coarser speckle pattern than a noncirrhotic liver (Yang et al. 1988). Moreover, the different speckle patterns resulting from different tissues may be utilized for image segmentation based on the textural approaches (Chen et al. 2000). Nevertheless, for many analysis tasks on the US images, the speckle basically acts as a noise because it deteriorates image quality, fine details and edge definition. It has been shown that the random mottle of the speckle degrades not only the perceivable resolution by a factor of

Address correspondence to: Chung-Ming Chen, Institute of Biomedical Engineering, College of Medicine, National Taiwan University, \#1, Sec. 1, Jen-Ai Road, Taipei, Taiwan. E-mail: ming@ lotus.mc.ntu.edu.tw five to seven (Kozma and Christensen 1976) but, also, the discriminability of subtle differences in grey levels (George et al. 1976).

To alleviate the negative effect of the speckle, two types of speckle-reduction approaches have been developed; namely, compounding and filtering. The compounding approaches attempt to eliminate the speckle by introducing incoherence during image formation ( $\mathrm{Li}$ and O'Donnel 1994; Quistgaard 1997). This is usually achieved by averaging a series of uncorrelated US images in the spatial or the frequency domain at the expense of a higher system complexity. These uncorrelated images may be sampled at different times, from different views, or with different frequencies for the same target.

Filtering approaches offer a practical alternative for most clinical applications. Although some filtering approaches reduce the speckle at a stage between logcompression and scan conversion (Abd-Elmonien et al. 2002; Thijssen 2003), most speckle-reduction algorithms estimate and eliminate the speckle solely using the displayed US images. This is because most clinical US image systems do not support any access to the logcompressed radiofrequency (RF) signals. 
An ultrasonic speckle-reduction algorithm usually comprises three components (i.e., speckle model, image model and despeckling strategy). The speckle model assumed in a speckle-reduction algorithm is the major component that distinguishes the despeckling processing from the conventional denoising processing. The speckle statistics have been correlated mainly with the effective scatterer density (i.e., the effective number of scatterers in a resolution cell of the tissue). The speckle is fully developed and the statistics of the echo envelope are Rayleigh distributed when the effective scatterer density is high (e.g., larger than 10) (Tuthill et al. 1988; Wanger et al. 1983). Except for the mean grey level, the first and the second order statistical characteristics of the fullydeveloped speckle are independent of tissue types (Thijssen and Oosterveld 1990). On the other hand, the speckle is partially developed and the echo envelope may be modeled by the $K$ distribution if the scatterer density is smaller than the Rayleigh limit (Jakeman and Tough 1987; Dutt and Greenleaf 1996).

The fully and the partially developed speckles on the displayed US images are governed by different distributions from the echo envelopes as a result of nonlinear postprocessing, such as log-transformation, interpolation, filtering and so on. The log-compressed fully developed speckle may be modeled by a double exponential or Fisher-Tippet density function, whereas the logcompressed partially-developed speckle may be approximated as a modified form of double exponential distribution by using Laguerre polynomials (Dutt and Greenleaf 1996).

Two other speckle models have also been considered previously. Jain (1989) presented a general multiplicative speckle model for the echo envelope, which was later adopted by Zong et al. (1998) and Achim et al. (2001). Loupas et al. (1989) suggested an empirical speckle model based on the observation that the mean is proportional to the variance rather than to the SD in a homogeneous region of US image. Kotropoulos (1994), Karaman et al. (1995), Hao et al. (1999) and Chen et al. (2003) have also employed this empirical model in formulating their speckle-reduction algorithms.

The image model characterizes the intensity variation of adjacent pixels in a displayed US image, which serves as the basis in the design of the criteria to gather pixels. Some widely used image models for general image processing are the flat-facet model, the slope-facet model and the piecewise-polynomial model (Haralick and Watson 1981; Koivunen 1995). For speckle reduction, most previous approaches have implicitly assumed the flat-facet model. The flat-facet model assumes that the uncorrupted image is composed of horizontal facets and the edges between adjacent facets are step edges.
The despeckling strategy, which is the core of a speckle-reduction algorithm, aims to attain an estimate, that is as close to the uncorrupted image (i.e., the image without being corrupted by the speckle) as possible. Depending on the despeckling strategy, most previous speckle reduction algorithms may be categorized into two classes of approaches; namely, filter-based approaches and wavelet-based approaches. Filter-based approaches count on the local statistics estimated from the displayed US image to eliminate the speckle at each pixel. For each pixel to be despeckled, called the target pixel, the local statistics are computed from a neighborhood of the target pixel, called the despeckling window. Generally speaking, the shape of the despeckling window may be regular or irregular. The shape and the size of the despeckling window may adaptively vary with the target pixel subject to specific criteria, or may be fixed for all target pixels. Some examples of filter-based approaches are the adaptive-weighted median filter (AWMF) (Loupas et al. 1989), the signal-adaptive maximum likelihood filter (SAMLF) (Kotropoulos 1994), the adaptive speckle suppression (ASSF) (Karaman et al. 1995), the adaptive speckle reduction filter (ASRF) (Dutt and Greenleaf 1996) and the 2-D weighted SavitzkyGolay filter (Chinrungrueng and Suvichakorn 2001).

One common deficiency of the previous filter-based approaches is the implicit assumption of the flat-facet image model. Although the flat-facet model allows a simple and fast despeckling strategy, it is unrealistic because the assumed horizontal facets and step edges are rarely found in the displayed US images. In reality, the ultrasonic image edges generally appear as a composition of linear or nonlinear ramps with various inclinations, which are due to the inherent point-spread function of the scanner and the inhomogeneous tissue property. The despeckling strategies based on the over-simplified flat facet model tend to distort the structures that are not linear or those where the local extrems occur.

The wavelet-based approaches decompose a displayed US image into multiscale images carrying information of different resolutions and attempt to remove the speckle ingredients separately at each scale. For examples, Zong et al. (1998) applied wavelet-shrinkage techniques based on multiscale soft thresholding to eliminate the speckle. Hao et al. (1999) separated the image into two parts by using the AWMF (Loupas et al. 1989) and both parts were recombined after despeckling. The speckle ingredients in each part were removed by using the wavelet analysis with a multiscale soft thresholding method modified from the Donoho's approach (Donoho 1995). Achim et al. (2001) modeled the subband decomposition of an US image as the alpha-stable distribution and designed a Bayesian estimator for statistically optimal signal extraction and speckle suppression. Macey 
and Page (2002) performed a standard noise-reduction technique, median filtering, in the wavelet domain.

One problem of the previous wavelet-based speckle reduction-algorithms is the lack of a reasonably good model to characterize the speckle ingredients in each image resolution. For example, Macey and Page (2002) simply applied median filtering to remove the speckle ingredients in the wavelet domain. Zong et al. (1998) and Hao et al. (1999) used only the empirical thresholding techniques to eliminate the speckle ingredients in each resolution, without statistical justification. Although Achim et al. (2001) showed that the signal components of the detailed signals at each scale might be modeled by the symmetrical alpha-stable distribution, the assumption that the noise was additive after the logarithmic transformation was not correct, especially in a nonflat area. Another problem is the inflexibility in adaptively changing the shape of the window for estimating the statistics of the signal and the speckle ingredients at each target pixel. Ideally, the shape of the analysis window should vary with the target pixel to account for the spatially variant nature of the speckle.

To preserve the higher order structures and to capture the spatially/variant property of the speckle, a new adaptive speckle-reduction algorithm, called the symmetrical speckle reduction filter (SSRF), was developed based on the slope-facet model. The basic idea of the SSRF was to estimate the uncorrupted signal on the largest symmetrical slope facet centered at each target pixel, which was determined by the region-growing technique. Although the slope-facet model is not the optimal model to describe the complex structure of US image, it is a reasonable choice, taking into account the implementation complexity and the closeness of the model to the surface profiles of the natural images. The symmetry constraint imposed on the slope facet ensured the correctness of the mean value. To find the largest symmetrical slope facet for each target pixel, two slope-similarity criteria for region growing were proposed to gather the pixels with similar slopes. These slope-similarity criteria were based on the local gradient and the ratio of the local variance to the mean (Loupas et al. 1989; Karaman et al. 1995). The local gradient tended to give a more accurate estimation of the slope, whereas the ratio of the local variance to the mean was less sensitive to the noise.

The proposed SSRF was compared with three previous speckle reduction algorithms, namely, the AWMF (Loupas et al. 1989), the ASSF (Karaman et al. 1995) and the Bayesian wavelet approach (Achim et al. 2001). The performance was evaluated using two types of images. One was a synthetic image with the simulated speckles and the other was a clinical US image. The synthetic image served for exact evaluation of the cor- rectness of the despeckled images because the uncorrupted images were known. The clinical US images were used only for visual inspection.

\section{MATERIALS AND METHODS}

The general approach of the filter-based specklereduction algorithms is to estimate the uncorrupted signal of a target pixel based on the local statistics of the despeckling window. By varying the shape and the size of the despeckling window, the filter-based algorithms have the great advantage of characterizing the spatiallyvariant property of the speckle. Nevertheless, the complex surface of the uncorrupted image demands an image model more realistic than the flat-facet model that had been implicitly assumed in the previous filter-based approaches.

As a filter-based speckle-reduction algorithm, the proposed SSRF has been designed with four essential ideas. The first idea was to model the image as a composition of the slope facets, rather than the flat facets for a more accurate estimation. The second idea was to incorporate the Loupas et al. (1989) empirical speckle model into the process of identifying the pixels on the same slope facet to account for the nature of the speckle in an US image. This empirical model was chosen because it could be easily generalized to characterize the speckle on a slope facet and, more importantly, it has been considered to be an acceptable model in several speckle-reduction algorithms (Kotropoulos 1994; Karaman et al. 1995; Hao et al. 1999; Chen et al. 2003). To ensure an unbiased estimation, the third idea was to use adaptive symmetrical despeckling windows, the shape and the size of which vary with the target pixel. It is easy to show that, if the despeckling window is not symmetrical, the mean value will not be correct, even if it is noise-free within the despeckling window. To utilize as much pixel information as possible for a statistically better estimation, the fourth idea was to use the two-stage despeckling process to remedy the potential restriction on finding the maximal possible despeckling window owing to the symmetry constraint. To describe the SSRF, the notations used throughout this paper are summarized as follows:

$f(p, q) \quad$ the intensity of the pixel $(p, q)$ in the displayed ultrasound image $f$ :

$f_{1}(p, q) \quad$ the intensity of the pixel $(p, q)$ in $f_{1}$, which is the image derived after the first-stage despeckling;

$f_{2}(p, q) \quad$ the intensity of the pixel $(p, q)$ in $f_{2}$, which is the final image derived after the second-stage despeckling;

$s(p, q) \quad$ the uncorrupted signal portion of the pixel $(p, q)$; 
$W_{s l}(x, y) \quad$ the largest symmetrical despeckling window for each target pixel $(x, y)$ in the first stage;

$W_{s 2}(x, y) \quad$ the largest symmetrical despeckling window for each target pixel $(x, y)$ in the second stage;

$W_{m}(p, q) \quad$ the $(2 m+1) \times(2 m+1)$ window centered at each pixel $(p, q)$;

$E(\phi(p, q)) \quad$ the expectation of $\phi(\cdot)$ at the pixel $(p, q)$; $\bar{E}(\phi(\mathrm{p}, \mathrm{q}, \mathrm{W}))=\frac{1}{|W(p, q)|} \sum_{(i, j) \epsilon W(p, q)} \phi(i, j)$, where $W(p$, $q)$ is any specified window centered at the pixel $(p, q)$ and $|W(p, q)|$ denotes the number of pixels in $W(p, q)$;

$G_{m 1}(p, q) \quad$ the gradient computed from $f$ using the window $W_{m}(p, q)$ for the pixel $(p, q)$ in the first stage;

$G_{m 2}(p, q) \quad$ the gradient computed from $f_{1}$ using the window $W_{m}(p, q)$ for the pixel $(p, q)$ in the second stage;

$$
\begin{gathered}
\mu_{m}(p, q)=\bar{E}\left(f\left(p, q, W_{m}\right)\right) ; \\
\sigma_{m}^{2}(p, q)=\bar{E}\left(\left(f\left(p, q, W_{m}\right)-\mu_{m}(p, q)\right)^{2}\right) ; \\
V M_{m}(p, q)=\sigma_{m}^{2}(p, q) / \mu_{m}(p, q) .
\end{gathered}
$$

The speckle model of Loupas et al. (1989) stated that the displayed US image might be modeled as:

$$
f(x, y)=s(x, y)+s^{1 / 2}(x, y) n(x, y),
$$

where $n$ was the random noise. The noise $n$ was assumed to be Gaussian white, ergodic and independent of the uncorrupted image $s$ (Kotropoulos 1994). The mean and the variance of the noise $n$ were assumed to be 0 and $\sigma_{n}^{2}$. This empirical signal-dependent speckle model was suggested based on the observation that, for a sufficiently large window (e.g., an $11 \times 11$ window), the ratio of the variance to the mean was approximately equal to a constant on a uniform area of a displayed US image. Based on the Loupas et al. speckle model, the proposed SSRF may be described as follows.

\section{The symmetrical speckle reduction filter}

Stage 1:

1. Derive the variance-to-mean map, $V M_{m}(x, y)$, by computing the ratio of the variance $\sigma_{m}^{2}(x, y)$ to the mean $\mu_{m}(x, y)$ of the $(2 m+1) \times(2 m+1)$ window centered at each pixel $(x, y)$. Derive the gradient map, $G_{m l}(x, y)$, by using the horizontal and vertical Kirsch gradient operators (Kirsch 1971).
2. For each target pixel, find the largest despeckling window by performing region-growing subject to the symmetry constraint and two similarity criteria (i.e., the gradient criterion and the variance-to-mean criterion).

3. Compute the mean of the pixel intensities within the despeckling window for each target pixel and replace the intensity of the target pixel by the mean.

Stage 2:

4. Derive the gradient map, $G_{m 2}(x, y)$, by using the horizontal and vertical Kirsch gradient operators (Kirsch 1971).

5. For each target pixel, find the largest despeckling window by performing region-growing subject to the gradient criterion only.

6. Compute the mean of the pixel intensities within the despeckling window for each target pixel and replace the intensity of the target pixel by the mean.

In both stages, the kernel process of the SSRF was to determine the largest symmetrical despeckling window containing the neighboring pixels on the same slope facet for each target pixel $(x, y)$. The kernel process was accomplished by using the region-growing scheme subject to the slope-similarity criteria. For the first stage, the slope-similarity criteria included the gradient criterion and the variance-to-mean criterion. More specifically, a pair of pixels, $\left(x_{i}, y_{i}\right)$ and $\left(x_{j}, y_{j}\right)$ that were symmetrical with respect to the target pixel, $(x, y)$, were included in the despeckling window only if the gradient criterion and the variance-to-mean criterion were satisfied simultaneously. For the second stage, because the speckle noise had been largely removed and the residual speckle noise could no longer be described by the Loupas et al. (1989) speckle model, only the gradient criterion was used as the slope- similarity criterion. The gradient criterion and the variance-to-mean criterion were defined as:

1. The gradient criterion for the stage $k, k=1$ or 2 :

$$
\left|G_{m k}(x, y)-G_{m k}\left(x_{i}, y_{j}\right)\right| \leq T_{G k}
$$

and

$$
\left|G_{m k}(x, y)-G_{m k}\left(x_{j}, y_{j}\right)\right| \leq T_{G k},
$$

2. The variance-to-mean criterion for the stage 1:

$$
\left|V M_{m}\left(x_{i}, y_{i}\right)-\frac{V M_{m}(x, y)+1-\gamma\left(c_{i}-1\right) \sigma_{n}^{2}}{c_{i}}\right| \leq T_{V M}
$$

and 


$$
\begin{gathered}
\mid V M_{m}\left(x_{i}, y_{j}\right)-\frac{V M_{m}(x, y)+(1-\gamma)\left(c_{j}-1\right) \sigma_{n}^{2}}{c_{j}} \\
\mid \leq T_{V M},
\end{gathered}
$$

where $\gamma=1 /(2 \mathrm{~m}+1)^{2}, T_{G k}$ and $T_{V M}$ are predefined thresholds. $c_{i}$ and $c_{j}$ are two constants satisfying $\mu_{m}\left(x_{i}\right.$, $\left.y_{i}\right)=c_{i} \mu_{m}(x, y)$ and $\mu_{m}\left(x_{j}, y_{j}\right)=c_{j} \mu_{m}(x, y) . \sigma_{n}{ }^{2}$ may be estimated from a uniform area.

For both the gradient $G_{m k}(p, q), k=1$ or 2 , and the variance-to-mean $V M_{m}(p, q)$, the size of $W_{m}(p, q)$ has been empirically set to $11 \times 11$ as a compromise between the statistical requirement of a constant varianceto-mean on a uniform area and the desire to have a spatial resolution that is as high as possible. The gradient $G_{m k}(p$, $q), k=1$ or 2 , was computed by using the horizontal and vertical Kirsch gradient operators (Kirsch 1971), which have been chosen only for their simplicity and variety of window sizes. One may use any other gradient estimator to derive a better estimation of the gradient for each pixel. For an image $g$, the horizontal and vertical Kirsch operators of size $(2 k+1) \times(2 k+1)$, denoted as $\Delta_{h}^{k}(\cdot)$ and $\Delta_{v}^{k}(\cdot)$, were defined as:

$$
\begin{array}{r}
\Delta_{h}^{k}(g(p, q))=\bar{E}\left(g\left(p-k / 2, q, W_{k}^{l}\right)\right)-\bar{E}(g(p \\
\left.\left.+k / 2, q, W_{k}^{r}\right)\right) \\
\Delta_{v}^{k}(g(p, q))=\bar{E}\left(g\left(p, q-k / 2, W_{k}^{d}\right)\right)-\bar{E}(g(p, q \\
\left.\left.+k / 2, W_{k}^{u}\right)\right)
\end{array}
$$

where $W_{k}^{l}, W_{k}^{r}, W_{k}^{d}$ and $W_{k}^{u}$ stood for the left, right, lower and upper halves of the window $W_{k}(p, q)$, respectively. These four subwindows did not contain the pixel $(p, q)$.

The gradient criterion has been devised to ensure that every pair of pixels, $\left(x_{i}, y_{i}\right)$ and $\left(x_{j}, y_{j}\right)$, included in the despeckling window are on the same slope facet as the target pixel $(x, y)$. On the other hand, the varianceto-mean criterion has been designed based on the variance relation between any two pixels, $(x, y)$ and $\left(x^{\prime}, y^{\prime}\right)$, on the same slope facet, which may be expressed as

$$
\begin{aligned}
\sigma_{m}^{2}\left(x^{\prime}, y^{\prime}\right) \approx \sigma_{m}^{2}(x, y)+(1-\gamma)(c & \\
& -1) \mu_{m}(x, y) \sigma_{n}^{2},
\end{aligned}
$$

where $s\left(x^{\prime}, y^{\prime}\right)=c s(x, y)$ and $\gamma=1 /(2 m+1)^{2}$. The derivation of the variance relation is provided in Appendix 1. Based on the variance relation, it is reasonable to impose the variance-to-mean criterion to examine whether or not two pixels are on the same slope facet.

After the largest symmetrical despeckling window
$W_{s k}(x, y), k=1$ or 2 , for a target pixel $(x, y)$ had been obtained in the stage $k$, the estimate for the target pixel in this stage was computed by taking the simple average of the pixel intensities within the window. To examine the correctness of the estimate, consider the mean value derived in the first stage for a target pixel $(x, y)$ :

$$
\begin{aligned}
f_{1} & (x, y)=\bar{E}\left(f\left(x, y, W_{s 1}\right)\right) \\
& =\bar{E}\left(s\left(x, y, W_{s 1}\right)\right)+\bar{E}\left(s^{1 / 2}\left(x, y, W_{s 1}\right) n\left(x, y, W_{s 1}\right)\right) \\
& =s(x, y)+\bar{E}\left(s^{1 / 2}\left(x, y, W_{s 1}\right) n\left(x, y, W_{s 1}\right)\right) \\
& =s(x, y)+\eta_{1}(x, y)
\end{aligned}
$$

where $\eta_{1}(x, y)=\bar{E}\left(s^{1 / 2}\left(x, y, W_{s 1}\right) n\left(x, y, W_{s I}\right)\right)$ represents the residual speckle noise at the end of the first stage. It can be shown that the mean and the variance of $\eta_{1}(x, y)$ are 0 and $s(x, y) \sigma_{n}{ }^{2} /\left|W_{s l}(x, y)\right|$, respectively. Note that $\bar{E}\left(s\left(x, y, W_{s 1}\right)\right)=s(x, y)$, because all pixels in $W_{s 1}$ are on the same slope facet and $W_{s I}$ is symmetrical with respect to $(x, y)$. Similarly, the mean value derived in the second stage (i.e., the final estimate obtained by the SSRF) for a target pixel $(x, y)$ can be shown to be:

$$
\begin{aligned}
f_{2}(x, y) & =\bar{E}\left(f_{1}\left(x, y, W_{s 2}\right)\right) \\
& =\bar{E}\left(s\left(x, y, W_{s 2}\right)\right)+\bar{E}\left(\eta_{1}(x, y)\right) \\
& =s(x, y)+\eta_{2}(x, y) \\
& \approx s(x, y)
\end{aligned}
$$

where $\eta_{2}(x, y)=\bar{E}\left(\eta_{1}(x, y)\right)$ represents the residual speckle noise in the final estimate. It can be shown that the mean and the variance of $\eta_{2}(x, y)$ are 0 and $s(x$, $y) \sigma_{n}{ }^{2} /\left(\left|W_{s 1}(x, y)\right|\left|W_{s 2}(x, y)\right|\right)$, respectively. When $\left(\mid W_{s 1}(x\right.$, $\left.y)|| W_{s 2}(x, y) \mid\right)$ is sufficiently large, the final estimate for the target pixel $(x, y)$ will be approximately equal to $s(x$, $y)$. Because $\left(\left|W_{s 1}(x, y)\right|\left|W_{s 2}(x, y)\right|\right)$ usually ranges from several 1000 s to $10 \mathrm{~s}$ of $1000 \mathrm{~s}$, it suggests that the proposed SSRF produces a reasonable estimate in most cases.

The large $\left(\left|W_{s l}(x, y)\right|\left|W_{s 2}(x, y)\right|\right)$ may be accredited to the two-stage despeckling process. From the viewpoint of information utilization, the two-stage despeckling process has utilized information much richer than can be provided by a single despeckling window. As a matter of fact, the actual number of pixels involved in computing the final estimate is much larger than the sizes of both despeckling windows. Moreover, even though the despeckling windows in both stages must be symmetrical, the actual region formed by all pixels involved in deriving the estimate for each target pixel may be asymmetrical. 
Comparative performance analysis

For comparative purposes, the proposed SSRF was compared with three previous speckle reduction algorithms, namely, the AWMF (Loupas et al. 1989), the ASSF (Karaman et al. 1995) and the Bayesian wavelet approach (Achim et al. 2001). The AWMF represented the class of filter-based speckle reduction algorithms with a filter of fixed shape and size. Let $W_{a}{ }^{A}(x, y)$ be the despeckling window of the target pixel $(x, y)$, the size of which was $(2 a+1) \times(2 a+1)$. Then the weight for the pixel $(i, j)$ in $W_{a}^{A}(x, y)$ was defined as:

$$
w_{a}^{A}(i, j)=w_{0}-\kappa \sqrt{(i-x)^{2}+(j-y)^{2}} V M_{a}(x, y),
$$

where $w_{0}$ was the constant weight for the center pixel of the despeckling window and $\kappa$ was a scaling constant. The basic idea of the AWMF was to assign a smaller weight to a pixel that was farther away from the center of the despeckling window. Moreover, if the target pixel was in a fast-varying area like a boundary area, because $V M_{a}(x, y)$ would be larger than that in a uniform area with the same local mean, the despeckling window was expected to have higher weight ratios (i.e., $w_{a}^{A}(x, y) / w_{a}^{A}(i$, $j), \forall(i, j) \in W_{a}^{A}(x, y)$ and $\left.(i, j) \neq(x, y)\right)$, than those despeckling windows in a uniform area. It meant that the AWMF tended to smooth more in the uniform areas and preserve the intensities of the pixels in the boundary areas.

The ASSF represented the class of filter-based speckle-reduction algorithms with a variable shape and size. Like the proposed SSRF, the ASSF used the regiongrowing technique to find the despeckling window for each target pixel $(x, y)$. A pixel $(i, j)$ was included in the despeckling window if the pixel $(i, j)$ was connected to the target pixel $(x, y)$ and satisfied the following similarity criterion:

$$
\left|V M_{m}(i, j)-V M_{m}(x, y)\right|<\beta\left(V M_{m}(x, y)\right)
$$

and

$$
\sqrt{(x-i)^{2}+(y-j)^{2}} \leq D_{b}
$$

where $D_{b}$ was a constant so that the total number of pixels in the despeckling window was not greater than $(2 m+1)^{2}$. The similarity bound, $\beta(\cdot)$, was defined as:

$$
\beta\left(V M_{m}(x, y)\right)=a+b \exp \left(-c V M_{m}(x, y)\right)
$$

where $a, b$ and $c$ were predefined constants. After the despeckling window had been found, the estimate for the target pixel $(x, y)$ was the mean or median of the pixel intensities in the window. Neighboring pixels with similar estimates were further merged if they had sufficient numbers of pixels in their despeckling windows (refer to Karaman et al. 1995).

The Bayesian wavelet approach proposed by Achim et al. (2001) was adopted as a representative of the wavelet-based approaches. In this approach, the displayed US image was first log-transformed and decomposed into multiscale images using wavelet transformation. Each detail coefficient was considered as the sum of a signal $s$ and a noise $\xi$. The signal $s$ was modeled as a symmetrical alpha-stable $(S \alpha S)$ distribution with a zero location parameter. The noise $\xi$ was assumed to be Gaussian-distributed with a zero mean. The Bayesian estimates of the signal component $\hat{s}$ was given by:

$$
\hat{s}=\frac{\int P_{\xi}(\xi) P_{s}(s) s d s}{\int P_{\xi}(\xi) P_{s}(s) d s}
$$

where $P_{\xi}(\xi)$ and $P_{s}(s)$ were the probability density functions (pdfs) of the signal $s$ and the noise $\xi$. The characteristic functions of $P_{\xi}(\xi)$ and $P_{s}(s)$ were denoted as $\phi_{\xi}(\omega)$ and $\phi_{s}(\omega)$, respectively. The following definition was made:

$$
\phi_{d}(\omega)=\phi_{s}(\omega) \cdot \phi_{\xi}(\omega)
$$

where

$$
\begin{gathered}
\phi_{s}(\omega)=\exp \left(-\gamma_{\mathrm{s}} \mid \omega^{\alpha_{s}}\right), 1<\alpha_{s} \leq 2 \\
\phi_{\xi}(\omega)=\exp \left(-\frac{\sigma^{2}}{2}|\omega|^{2}\right) .
\end{gathered}
$$

To derive $P_{\xi}(\xi)$ and $P_{s}(s)$, the parameters $\alpha_{s}, \gamma_{s}$ and $\sigma$ were estimated by fitting $\Phi_{d}(\omega)$ with the Fourier transform of the empirical pdf of the detail coefficients $\Phi_{d_{e}}(\omega)$ in a least-squares sense.

\section{Image for performance analysis}

To evaluate the performances of the proposed SSRF and three previous approaches, two types of images were used in this study; namely, the synthetic images and the clinical US images. The clinical US images offered the real image condition to test these four speckle reduction algorithms. However, because the uncorrupted US images (i.e., the images without the speckle) were not known, the clinical US images could only be used for 


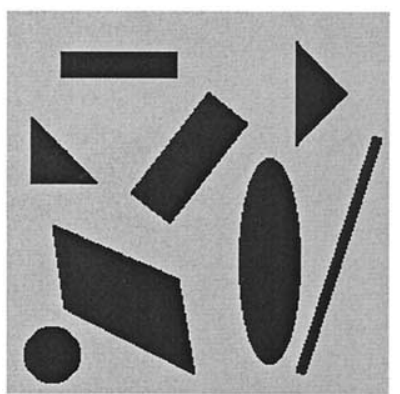

(a)

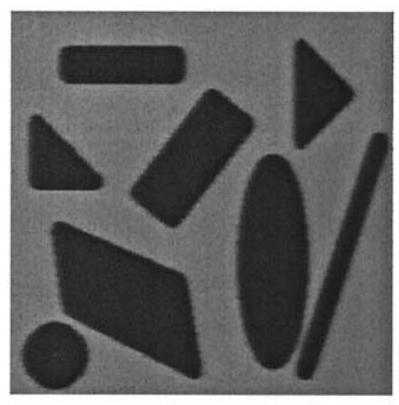

(c)

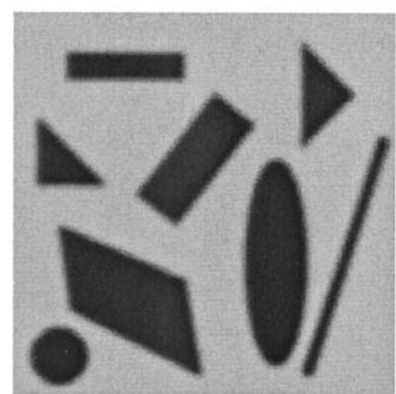

(b)

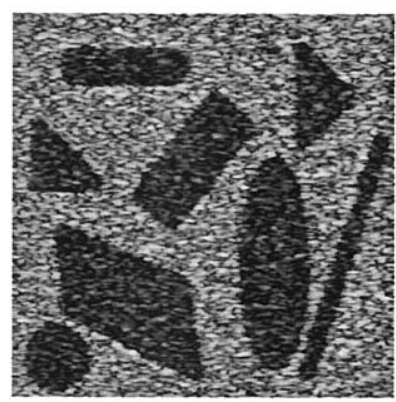

(d)
Fig. 1. (a) The raw synthetic image; (b) smoothed raw synthetic image with $\sigma_{g}=2.5$; (c) the blurred SRS image with $\sigma_{g}=2.5$, which was used as the ideal image for the image shown in (d); and (d) the synthetic image with the speckle, the $C N R$ of which was approximately 2.0 .

qualitative evaluation. On the other hand, because the synthetic images were generated through simulation, the quantitative performance analysis of this study was carried out mainly based on the synthetic images.

The synthetic images simulated the displayed US images composed of hypoechoic structures with sharp corners, ramp edges with various slopes and log-compressed fully developed speckles. The ramp edges were created by smoothing the raw synthetic image shown in Fig. 1a with zero-mean Gaussian functions of different SDs $\sigma_{g}$. For convenience, the smoothed raw synthetic image is abbreviated as SRS image throughout this paper. Figure $1 \mathrm{~b}$ demonstrates an SRS image with $\sigma_{g}=$ 2.5.

By using the SRS image, the log-compressed fullydeveloped speckle was simulated based on the speckle simulation approach used by Li and O'Donnell (1994). The speckle was modeled as random walks in the complex plan, each of which stood for the signal received by the transducer from a scatterer in the resolution volume. Based on the central limit theory, the real part and the imaginary part of the summed signal were assumed to be Gaussian-distributed, and therefore, the amplitude of the summed signal was Rayleigh-distributed. The scanner was constructed as a 128-element linear array with 0.25 $\mathrm{mm}$ interelement spacing. The central frequency and the band width were set to $3 \mathrm{MHz}$ and $1 \mathrm{MHz}$, respectively. The axial direction was along the vertical axis of the simulated image. For the axial response, the shape of the envelope was assumed to be Gaussian. The lateral response was the Fourier transform of the aperture function. The point-spread function of the scanner was assumed to be spatially invariant.

By controlling the intensities of the foreground and the background, the synthetic images with varieties of contrast-to-noise ratios $(C N R \mathrm{~s})$ were generated to evaluate the performances of these four speckle reduction algorithms. The $C N R$ was defined as:

$$
C N R=\frac{\left|g_{f}-g_{b}\right|}{\max \left(\sigma_{f}, \sigma_{b}\right)}
$$

where $\sigma_{f}$ and $\sigma_{b}$ were the SDs of the speckle in the foreground and the background areas, excluding the ramps. $g_{f}$ and $g_{b}$ were the mean intensities of the foreground and the background, respectively.

\section{Performance indices}

Speckle reduction is an essential process for two classes of US image analyses. One is feature-extraction of the uncorrupted image (e.g., boundary detection of the object-of- interest) and the other is visual lesion detection. For the former, speckle reduction aims to attain a despeckled image as close to the ideal image (i.e., the uncorrupted image) as possible, to ensure the correctness of the extracted features. For the latter, speckle reduction attempts to enhance the visual lesion detectability.

To account for the different emphases of these two classes, several performance indices have been used previously, such as the mean-square error (MSE) (Achim et al. 2001; Abd-Elmonien et al. 2002), the pooled signalto-noise ratio (SNR), the pooled CNR (Chen et al. 2003), the area of the receiver operating characteristic (ROC) curve for distinguishing the foreground and the background by thresholding (Rakotomamonjy and Marche 1998), the size preservation of a lesion defined by the derived boundaries of the object-of-interest (Chen et al. 1996) and so forth. The MSE measures how close the despeckled image is to the ideal image. Ideally, the closer the despeckled image is to the ideal image, the more likely that a correct feature for the object-of-interest may be derived. On the other hand, the last five indices quantify the lesion detectability based on different image properties. It should be noted that, except for the last one, all these indices provide global measures (i.e., they are computed based on the information of the entire image). One shortfall of these global measures is that they consider all pixels to be equally important when 
assessing the image quality. As a result, they may overlook local information around edges that, may be decisive for lesion detection. For example, it is not unusual that, even if the overall quality of the entire image is bad, one can still detect a lesion easily when the image quality around the lesion boundary is reasonably good. Although the last index does emphasize the local quality of the despeckled image around the lesion boundary, it requires a "gold standard" edge detector for a fair evaluation and this does not currently exist.

In this study, two performance indices were used to evaluate these four speckle-reduction algorithms. One served as the global measure and the other, as the local measure. The first performance index was the root-meansquared (RMS) difference between the ideal image and the despeckled image. The RMSD is basically the squared root of the MSE. The RMSD is also closely related to the SNR and CNRs of the despeckled image. Conceptually, the ideal image has the best SNR and CNRs that a speckle reduction algorithm can ever attain, provided that no operation other than speckle reduction is performed. As the despeckled image becomes more similar to the ideal image, which implies a smaller $R M S D$ the SNR and CNR tend to be higher. Therefore, the $R M S D$ not only indicates the global similarity between the ideal image and the despeckled image, but also carries the measure of overall lesion detectability of the despeckled image.

Let $X_{\mathrm{SSRF}}, X_{\mathrm{AWMF}}, X_{\mathrm{ASSF}}$ and $X_{\text {Wavelet }}$ denote the despeckled images obtained by using the SSRF, AWMF, ASSF and the Bayesian wavelet approaches, respectively. Also, $X_{C}$ denoted the synthetic image with the speckle. The $R M S D$ of an image $X_{S}, S \in\{\mathrm{SSRF}, \mathrm{AWMF}$, ASSF, Wavelet, $C$, denoted as $\operatorname{RMSD}\left(X_{S}\right)$, was defined as:

$$
\operatorname{RMSD}\left(X_{S}\right)=\sqrt{\frac{1}{M N} \sum_{i=1}^{N} \sum_{j=1}^{M}\left(X_{S}(i, j)-X_{0}(i, j)\right)^{2}}
$$

where $X_{0}$ stood for the corresponding ideal image of $X_{S}$, $X_{S}(i, j)$, the grey level of the pixel at $(i, j)$ in $X_{S}$ and $M N$, the size of the image.

It should be noted that the SRS image was degraded, not only by the speckle noise, but also by the blurring effect caused by the point-spread function of the scanner. Because the emphasis of this study was on despeckling, the blurred SRS image (by the point-spread function of the scanner) was used as the ideal image, rather than the SRS image itself. The blurred SRS image was generated in exactly the same way as the synthetic image with the speckle, except that the speckle noise was not added. Figure 1c shows the blurred SRS image with $\sigma_{g}=2.5$, which was used as the ideal image for the synthetic image with the speckle shown in Fig. 1d. The CNR of Fig. 1d was approximately 2.0.

To remedy the deficiency of the global measure inherent in the $R M S D$, the second performance index considered in this study attempted to provide an assessment of local lesion detectability. The second performance index, denoted by LLDI (local lesion detectability index), was a local version of the global performance index used by Rakotomamonjy and Marche (1998). In their work, the range between the maximum and the minimum of the grey levels of the despeckled image was divided into 100 levels. By using each of these 100 levels as a threshold, all pixels in the despeckled image were labeled as either foreground or background pixels. The ROC curve was then plotted for the sensitivity and (1-specificity) computed with these 100 thresholds. The area under the ROC curve was used as the performance index for assessment of the speckle-reduction algorithms. To incorporate the notion of the local measure, the $L L D I$ used only the pixels around the boundaries of the objects of interest to derive the ROC curve, instead of using all pixels as used by Rakotomamonjy and Marche (1998). More specifically, the LLDI was defined as the area of the ROC curve computed for the pixels on the ramps around the boundaries of the objects of interest specified by the ideal image.

\section{RESULTS AND DISCUSSIONS}

To evaluate the performances of these four approaches on various image conditions, 12 sets of images, each characterized by a representative combination of $\left(C N R, \sigma_{g}\right)$, were employed in this study, where $C N R \epsilon$ $\{1,3,5\}$ and $\sigma_{g} \epsilon\{0.5,1.5,2.5,3.5\}$. To reduce the sample bias in the random process of speckle simulation, each set of images consisted of five images with the same $\left(C N R, \sigma_{g}\right)$.

The vertical bars in Fig. 2 show the mean RMSDs of the original images (i.e., the synthetic images with the speckle and the despeckled images derived by the SSRF) which are labeled as "Original" and "SSRF", respectively. In addition, the line curve in Fig. 2, labeled as (Original-SSRF)/Original, indicates the average reduction rates for the $R M S D$ achieved by the SSRF, which were defined as:

$$
\frac{\left(R M S D\left(X_{C}\right)-R M S D\left(X_{S S R F}\right)\right)}{R M S D\left(X_{C}\right)} \times 100 \%
$$

where $R M S D\left(X_{s}\right)$ stood for the $R M S D$ of the image $X_{s}$. Similarly, Fig. 3 illustrates the mean LLDIs of the original images and the images despeckled by the SSRF, as well as the average improvements on the LLDIs achieved 


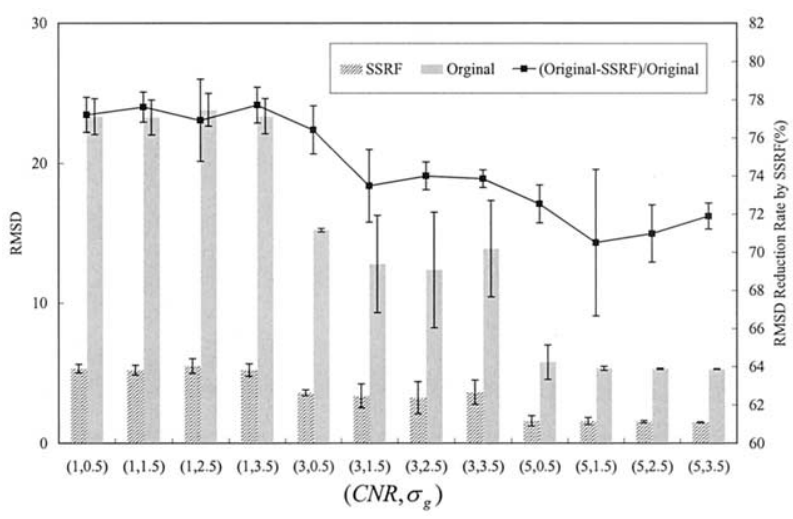

Fig. 2. Vertical bars: the mean RMSDs of the original images and the despeckled images derived by the SSRF, labeled = "Original" and "SSRF," respectively. Line curve: the average reduction rates for the $R M S D$ achieved by the SSRF, labeled = (Original-SSRF)/Original.

by the SSRF, which were defined as:

$$
\frac{\left(L L D I\left(X_{S S R F}\right)-L L D I\left(X_{C}\right)\right)}{\operatorname{LLDI}\left(X_{C}\right)} \times 100 \%
$$

where $L L D I\left(X_{s}\right)$ represented the $L L D I$ of the image $X_{s}$. The error bars in both Figs. 2 and 3 denote one SD of the $R M S D$ s or the LLDIs for each set of images.

Figures 2 and 3 clearly show that the SSRF could significantly improve the RMSDs and the LLDIs over the original images for the synthetic images simulated in this study. Most $R M S D$ reduction rates were higher than $70 \%$ and the improvements on the LLDIs were mostly greater than $4 \%$. To further validate the significance of the

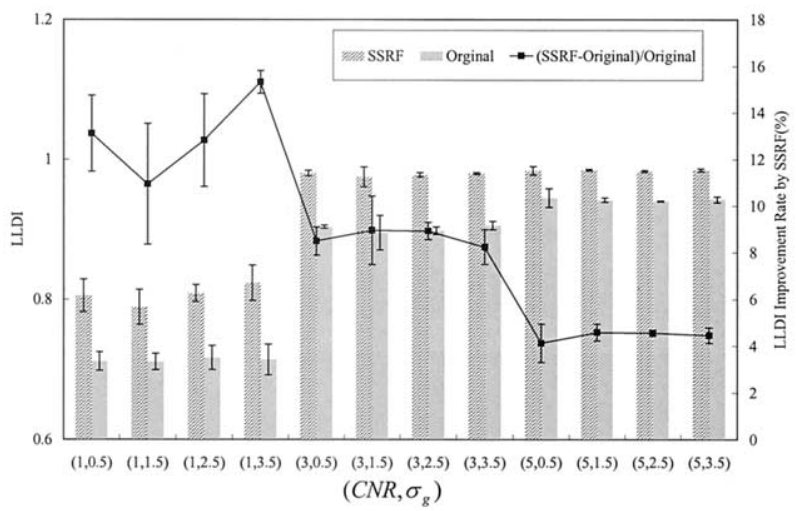

Fig. 3. Vertical bars: the mean $L L D I$ s of the original images and the despeckled images derived by the SSRF, labeled = "Original" and "SSRF", respectively. Line curve: the average improvement rates on the $L L D I$ achieved by the SSRF, labeled = (SSRD-Original)/Original.

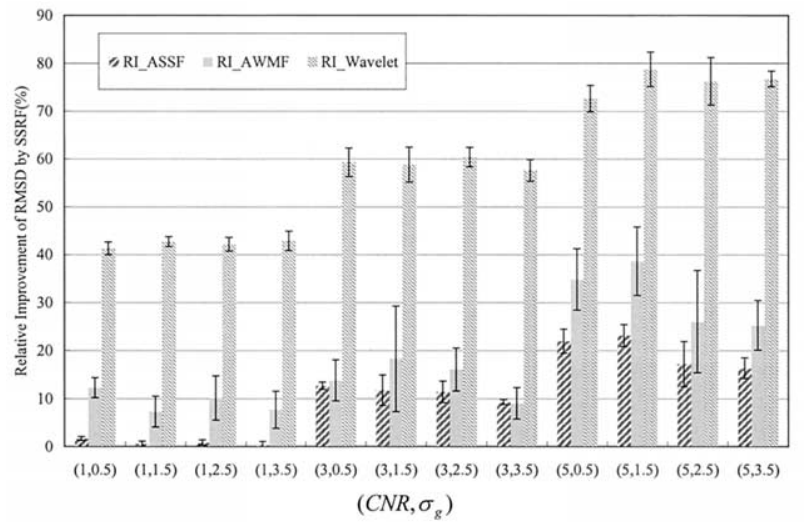

Fig. 4. The improvements made by the SSRF relative to the RMSDs attained by the AWMF, the ASSF and the Bayesian wavelet approaches, labeled $=$ AWMF, ASSF and Wavelet, respectively.

improvements, for each set of images, the single-tailed paired-sample $t$-test was used to test the null hypothesis that the RMSDs and the LLDIs of the images despeckled by the SSRF were not better than those of the original images, respectively. The significance level was set to $5 \%$. The test results showed that the $p$ values for all 24 comparisons (12 for $R M S D$ s and 12 for $L L D I \mathrm{~s}$ ) were smaller than 0.001. It suggested that all null hypotheses be rejected and, hence, we concluded that the RMSDs and the LLDIs achieved by the SSRF were significantly better than those of the original images at the 5\% significance level for each set of images.

Furthermore, Figs. 2 and 3 indicate that the $C N R$ was the more influential factor than the $\sigma_{g}$ in determining the RMSDs and the $L L D I$ s achievable by the proposed SSRF. As can be seen from Figs. 2 and 3, the mean $R M S D$ s and $L L D I$ s achieved by the SSRF were roughly the same for the image sets with the same $C N R$, whereas they were improved as the $C N R$ increased. Relative to the original images, the proposed SSRF tended to achieve a larger improvement for an image with a smaller $C N R$.

In comparison with the AWMF, the ASSF and the Bayesian wavelet approaches, for the $R M S D$, the relative improvements achieved by the SSRF over each of these three approaches $S, S \in\{\mathrm{AWMF}$, ASSF, Wavelet\}, are provided in Fig. 4 and those for the LLDI are given in Fig. 5. For the $R M S D$, the relative improvement over the algorithm $S$, denoted as $R I \_S$ in Fig. 4, $S \in\{A W M F$, $A S S F$, Wavelet $\}$, was defined as:

$$
R I \_S=\frac{R M S D\left(X_{S}\right)-R M S D\left(X_{S S R F}\right)}{R M S D\left(X_{C}\right)-R M S D\left(X_{S}\right)} \times 100 \%
$$

Likewise, for the $L L D I$, the relative improvement over 


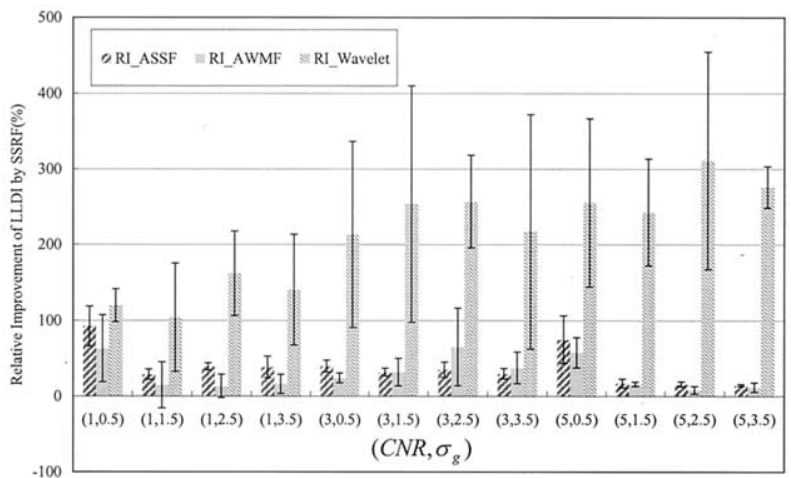

Fig. 5. The improvements made by the SSRF relative to the LLDIs attained by the AWMF, the ASSF and the Bayesian wavelet approaches, labeled = AWMF, ASSF and Wavelet, respectively.

the algorithm $S$, denoted as $R I \_S$ in Fig. 5, $S \in$ \{AWMF, ASSF, Wavelet\}, was defined as:

$$
R I \_S=\frac{L L D I\left(X_{S}\right)-\operatorname{LLDI}\left(X_{S S R F}\right)}{\operatorname{LLDI}\left(X_{C}\right)-\operatorname{LLDI}\left(X_{S}\right)} \times 100 \%
$$

The error bars in both Figs. 4 and 5 denote one SD of the $R I \_S$ values for each set of images.

In terms of the RMSD, Fig. 4 depicts that the proposed SSRF outperformed these three previous algorithms for all synthetic images simulated in this study. This observation was further validated by using the single-tailed paired-sample $t$-tests. The null hypothesis was that, for each set of images, the RMSDs achieved by the SSRF were not smaller than those attained by the algorithm $S, S \in\{A W M F, A S S F$, Wavelet $\}$. The significance level was set to $5 \%$. The test results showed that the $p$ values for all 36 comparisons (12 for each of these three previous algorithms) were smaller than 0.05 . It suggested that all null hypotheses be rejected and we concluded that the RMSDs achieved by the SSRF were significantly better than those obtained by the three previous algorithms at the 5\% significance level for each set of images.

Although Fig. 4 shows the superior performance of the SSRF in terms of the global measure, the RMSD, Fig. 5 reveals that, in terms of the local measure, the $L L D I$, the proposed SSRF was also better than its counterparts in all tested cases but two, that is when using the AWMF algorithm with $\left(C N R, \sigma_{g}\right)=(1,1.5)$ and $(1,2.5)$. To validate this observation, two kinds of paired-sample $t$-tests were carried out. For each of these two exceptions, that is, $\left(C N R, \sigma_{g}\right)=(1,1.5)$ and $(1,2.5)$, the two-tailed paired-sample $t$-test was used and the null hypothesis was that, for each set of images, the LLDIs achieved by the SSRF and the AWMF were statistically equivalent.
The significance level was set to $5 \%$. Because the $p$ values for both cases were larger than 0.1 , the null hypotheses were accepted and we concluded that, for each set of images, the SSRF and the AWMF were statistically equivalent in these two cases in terms of the $L L D I$. For each of the cases, other than these two exceptions, the single-tailed paired-sample $t$-test was used and the null hypothesis was that, for each set of images, the $L L D I$ s achieved by the SSRF were not larger than those attained by the algorithm $S, S \in\{A W M F, A S S F$, Wavelet $\}$. The significance level was set to $5 \%$. The test results showed that all $p$ values were smaller than 0.02 . It suggested that the null hypotheses be rejected and we concluded that, excluding these two exceptions, the $L L$ $D I$ s achieved by the SSRF were significantly better than those obtained by the three previous algorithms at the 5\% significance level for each set of images.

For the simulated synthetic images, the SSRF were shown to be superior to the AWMF, the ASSF and the Bayesian wavelet algorithm in terms of the RMSD and the LLDI. Moreover, for the RMSD, the SSRF tended to gain more improvement over these three approaches as the $C N R$ increased and, given a $C N R$, the improvements over the AWMF and the ASSF were inclined to decrease as $\sigma_{g}$ increased. Although the former was mainly due to the better gradient estimation attainable for a larger $C N R$, the latter might be attributed to the fact that, as $\sigma_{g}$ increased, the edges become flatter and the approximation error caused by the flat-facet model become smaller.

Among these three previous approaches, the Bayesian wavelet algorithm (Achim et al. 2001) had the worst performance in terms of both global and local measures. One possible reason for the worst performance of the Bayesian wavelet algorithm was the use of a global threshold for the entire image, which did not take into account the signal-dependent nature of that the speckle. Another reason was that the threshold proposed by Achim and colleagues did not work very well in practice. In this study, we tried to find a better threshold by considering all possible integral multiples of the thresholds derived according to the formula proposed in Achim et al. (2001).

Additionally, the ASSF generally performed better than the AWMF in terms of the RMSD, but had similar performance to that of the AWMF in terms of the LLDI. It was reasonable because the ASSF not only took into account the speckle statistics, but also enhanced the quality of averaging operations with the constraints inherently imposed by the region-growing technique. Nevertheless, because the conventional region-growing approach did not work well on a ramp, the local performance of the ASSF was not as impressive as its global performance. 


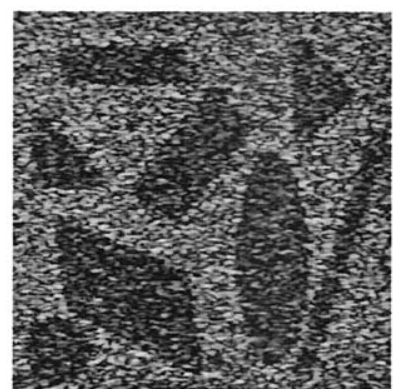

(a)

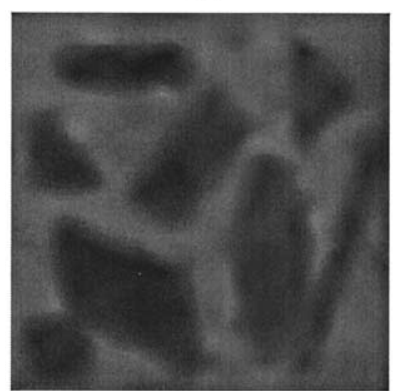

(c)

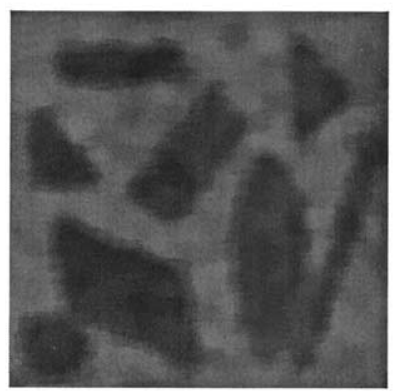

(e)

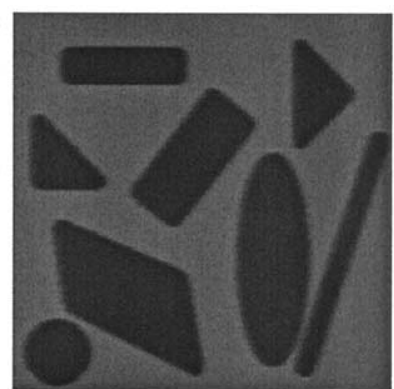

(b)

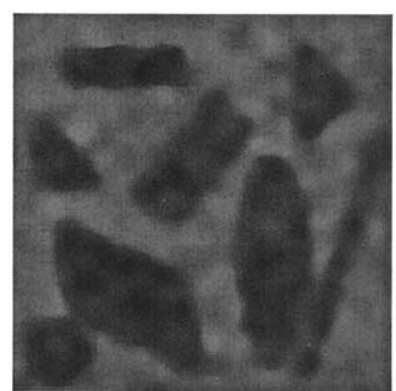

(d)

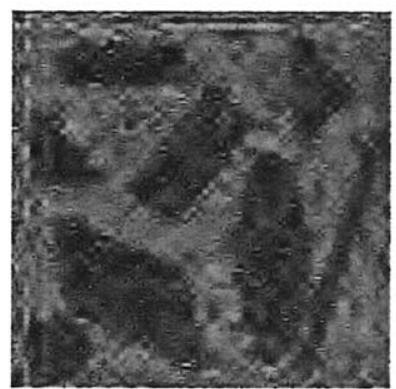

(f)

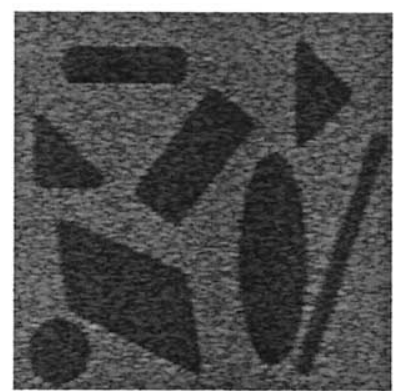

(a)

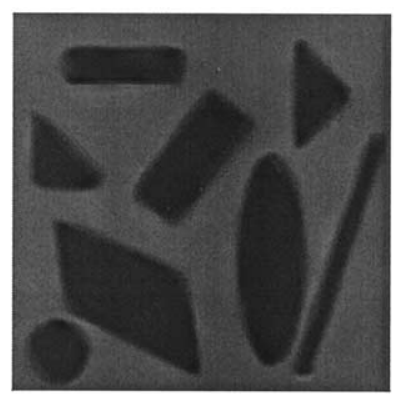

(c)

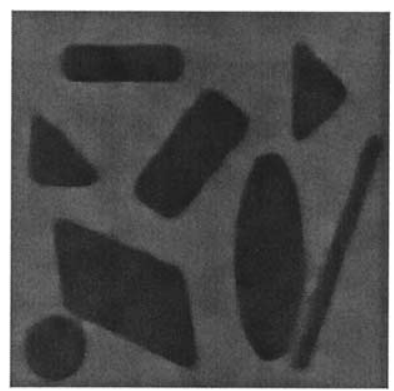

(e)

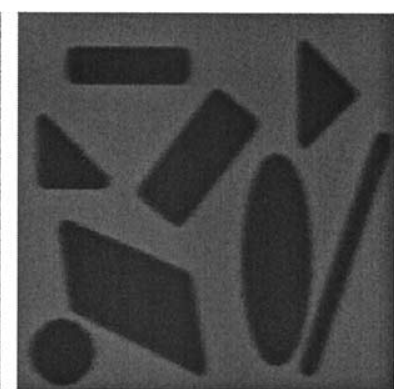

(b)

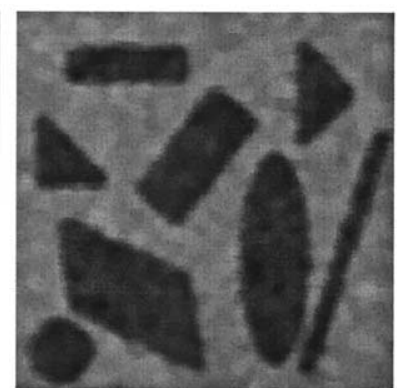

(d)

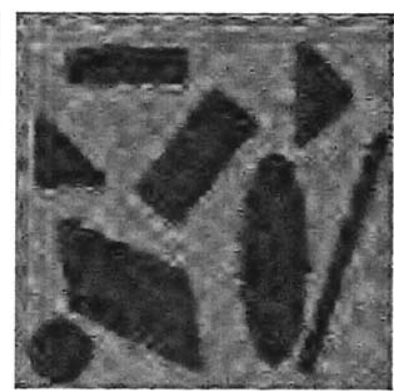

(f)
Fig. 6. (a) The synthetic image with the speckle, where $(C N R$, $\left.\sigma_{g}\right)=(1,2.5)$; (b) the ideal image of (a); (c) the despeckled image obtained by using the proposed SSRF; (d) the despeckled image obtained by using the AWMF; (e) the despeckled image obtained by using the ASSF; and (f) the despeckled image obtained by using the Bayesian wavelet approach.

For a visual comparison of the despeckled images derived by these four speckle reduction algorithms, Fig. 6 demonstrates the despeckled images for one case of $\left(C N R, \sigma_{g}\right)=(1,2.5)$. Figure $6 \mathrm{a}$ and $\mathrm{b}$ shows the synthetic image with the speckle and its ideal image, respectively. Figure $6 \mathrm{c}-\mathrm{f}$ presents the despeckled images derived by the SSRF, the AWMF, the ASSF and the Bayesian wavelet approaches, respectively. Likewise, Figs. 7 and 8 provide the synthetic images with the speckle, the ideal images and the despeckled images for $\left(C N R, \sigma_{g}\right)=$ $(3,2.5)$ and $(5,2.5)$, respectively. None of these four algorithms yielded impressive results for $\left(C N R, \sigma_{g}\right)=$
$(1,2.5)$, as shown in Fig. 6 . But, the SSRF gave a slightly smoother despeckled image than its counterparts.

When $\left(C N R, \sigma_{g}\right)=(3,2.5)$, the despeckled images attained by using the SSRF (Fig. 7c) and the ASSF (Fig. 7e) appeared more pleasing than the other two. Comparing Fig. $7 \mathrm{c}$ and e, one may find that the SSRF had preserved the corners of the objects better than the ASSF, which may be accredited to the symmetry constraint and the gradient criterion leading to a more accurate estimate. As $C N R$ increased to 5, all four algorithms produced reasonably good despeckled images, as displayed in Fig. 8. Nevertheless, the quality of the despeckled image derived by the 


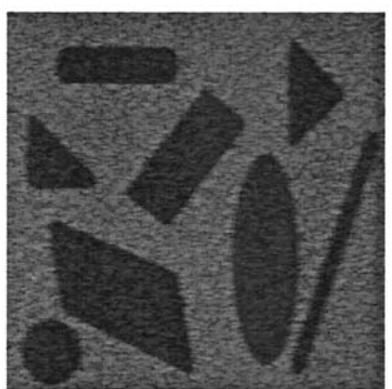

(a)

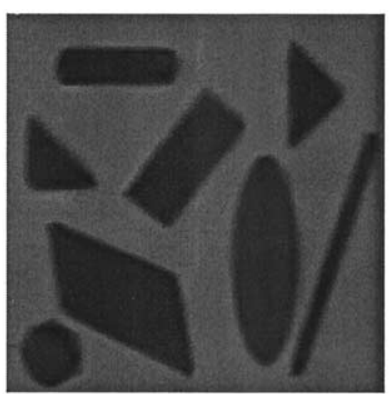

(c)

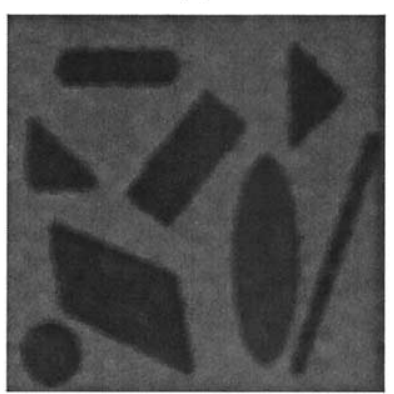

(e)

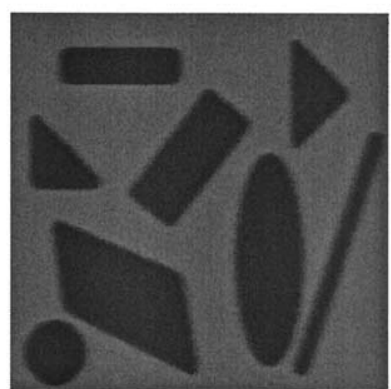

(b)

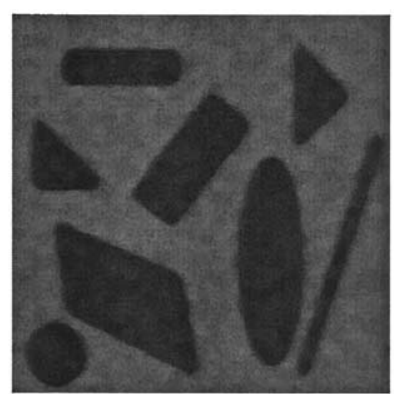

(d)

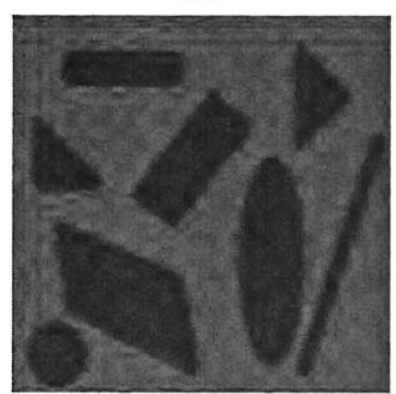

(f)

Fig. 8. (a) The synthetic image with the speckle, where $(C N R$, $\left.\sigma_{g}\right)=(5,2.5)$; (b) The ideal image of (a); (c) The despeckled image obtained by using the proposed SSRF; (d) The despeckled image obtained by using the AWMF; (e) the despeckled image obtained by using the ASSF; and (f) the despeckled image obtained by using the Bayesian wavelet approach.

SSRF was still superior to that of the other three images in terms of the image smoothness and the corner preservation.

To examine the performance of these four algorithms on the clinical US images, Figure 9a shows a clip of an image composed of two side-by-side liver US

Fig. 9. (a) A clip of an image composed of two side-by-side liver US images, each with a hemangioma; (b) The despeckled image obtained by using the proposed SSRF; (c) The despeckled image obtained by using the AWMF; (d) The despeckled image obtained by using the ASSF; and (e) The despeckled image obtained by using the Bayesian wavelet approach.

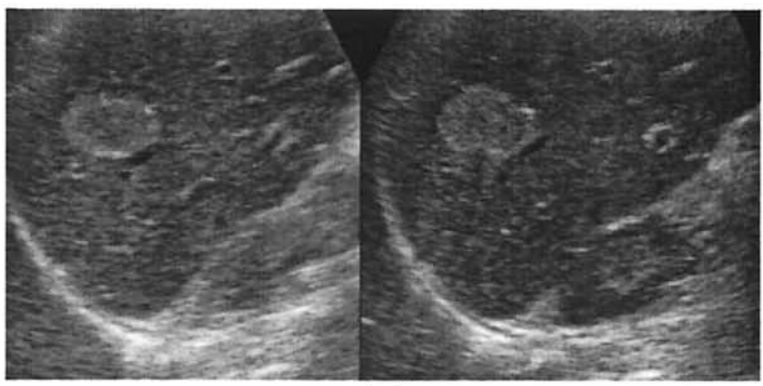

(a)

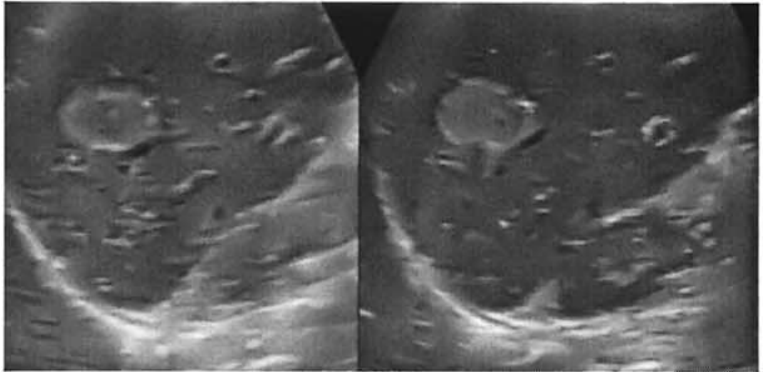

(b)

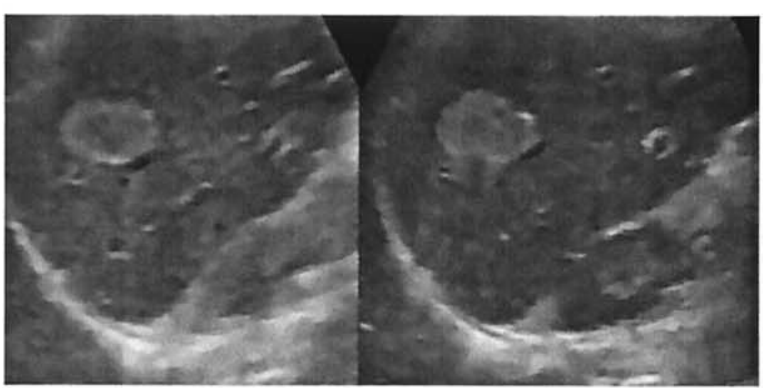

(c)

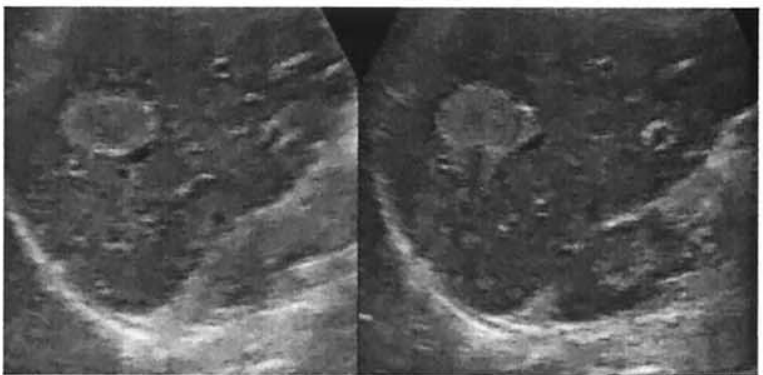

(d)

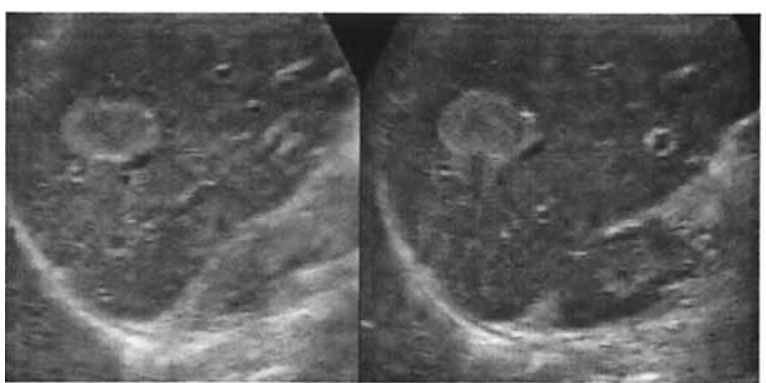

(e) 


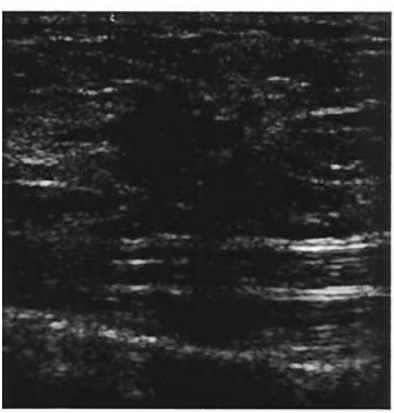

(a)

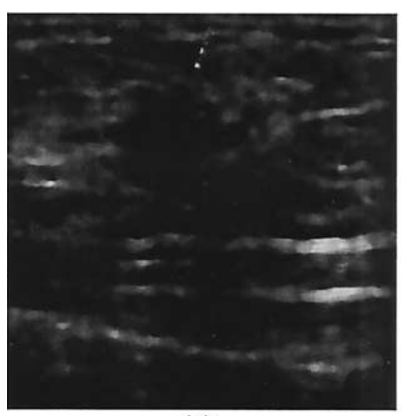

(c)

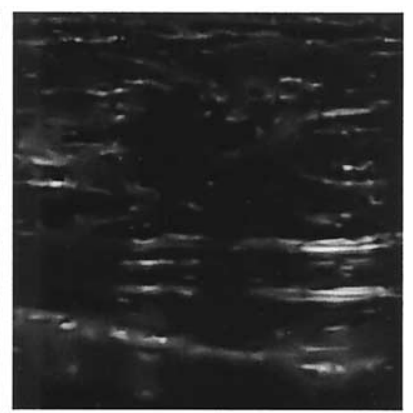

(b)

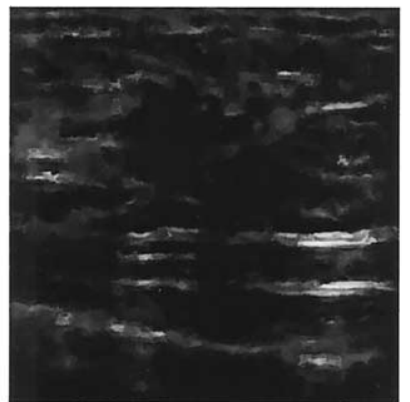

(d)

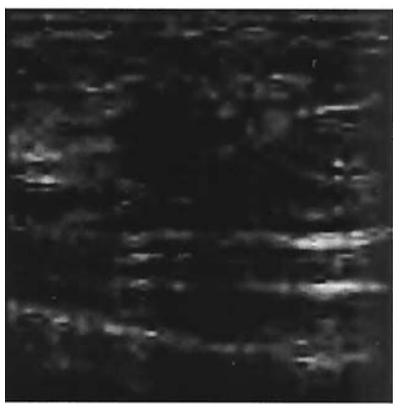

(e)

Fig. 10. (a) A clip of a breast US image with a malignant lesion in the middle of the upper half; (b) The despeckled image obtained by using the proposed SSRF; (c) The despeckled image obtained by using the AWMF; (d) The despeckled image obtained by using the ASSF; and (e) The despeckled image obtained by using the Bayesian wavelet approach.

images, each with a hemangioma. The image was captured from the RGB (Red-Green-Blue) outputs of a Toshiba SSA-380A clinical US imaging system (Toshiba Corporation, Tokyo, Japan) through the frame grabber card, Meteor-II card, made by the Matrox Electronic System Ltd. (Quebec, Canada). The operating frequency was $3 \mathrm{MHz}$. Figure 9b-e presents the despeckled images derived by using the SSRF, the AWMF, the ASSF and the Bayesian wavelet approaches, respectively. It is clear that the despeckled image obtained by the SSRF is much better than the other three images. More specifically, the despeckled image obtained by the SSRF not only looks smoother but, also, reveals more fine details than the other three images. For instance, the small lumps around the left hemangioma can be clearly seen in Fig. 9b, but not in the other three images.

As another example, Fig. 10a demonstrates a clip of a breast US image with a malignant lesion in the middle of the upper half. The image was directly stored (using the system built-in function) from an HDI 3000 US imaging system (Advanced Technological Laboratory; Bothell, WA), equipped with a broadband L10-5 linear electronically focused transducer and cine loop capability. Compared to the liver US image given in Fig. 9a, the breast US image in Fig. 10a was much noisier because of its larger grain size of the speckle and its worse defined edges. As in Fig. 9, Fig. 10b-e shows the despeckled images derived by using the SSRF, the AWMF, the ASSF and the Bayesian wavelet approaches, respectively. In this case, the SSRF has also attained a better image than the other three algorithms in terms of the image smoothness, fine details preservation and the edge definitions. For example, the patch effect is clearly seen in the image derived by using the ASSF and the fine details such as the bright spots in Fig. 10a have been largely smeared out by the AWMF and the ASSF. The image derived by using the Bayesian wavelet approach looked foggy and the boundary of the lesion was hardly differentiable. Among these four algorithms, the proposed SSRF was the most successful algorithm in removing the speckle within the lesion and the Bayesian wavelet approach was the worst.

\section{CONCLUSIONS}

To account for the complex nature of the ultrasonic image surfaces and the signal-dependency of the speckle, in this paper, we propose a new speckle reduction algorithm featuring the slope-facet model, the Loupas et al., (1989) empirical speckle model, the adaptive symmetric despeckling window and the twostage despeckling strategy. The slope-facet model allowed the proposed SSRF to estimate the ideal image more realistically than the flat-facet model, which had been implicitly or explicitly assumed by most previous speckle-reduction algorithms. The Loupas and colleagues' speckle model played an essential role in identifying the pixels in the same despeckling window with similar speckle statistics. The adaptive and the symmetrical properties of the despeckling window captured the locally variant statistics of the speckle and ensured the correctness of the mean on a slope facet, respectively. The two-stage despeckling strategy was employed to enhance the statistical reliability of each estimate by forming a union of a set of symmetrical despeckling windows, which not only provided a 
larger number of pixels for averaging but also practically relaxed the symmetry constraint.

The proposed SSRF clearly outperformed the AWMF, the ASSF and the Bayesian wavelet approaches in both of the synthetic images with the speckle and the clinical US images tested in this study. The experimental results showed that the proposed SSRF was able to reduce more than $70 \%$ of the RMSD and increase more than $4 \%$ of the LLDIs of the synthetic images with the speckle. The superiority of the SSRF compared with the other three was validated by paired-samples $t$-tests. Except in two cases, the test results suggested that the SSRF is better than the other three approaches in terms of the RMSD and the LLDI at the $5 \%$ significance level. Supported by the superior performance of the SSRF, it is suggested that the slope-facet model be used for speckle reduction, instead of the flat-facet model.

\section{REFERENCES}

Abd-Elmoniem KZ, Youssef ABM, Kadah YM. Real-time speckle reduction and coherence enhancement in ultrasound imaging via nonlinear anisotropic diffusion. IEEE Trans Biomed Eng 2002;49:9971014.

Achim A, Bezerianos A, Tsakalides P. Novel Bayesian multiscale method for speckle removal in medical ultrasound images. IEEE Trans Med Imaging 2001;20(8):772-783.

Chen CM, Lu HHS, Han KC. A textural approach based on Gabor functions for texture edge detection in ultrasound images. Ultrasound Med Biol 2000;27:515-534.

Chen Y, Broschat SL, Flynn PJ. Phase insensitive homomorphic image processing for speckle reduction. Ultrason Imaging 1996;18:122-139.

Chen Y, Yin R, Flynn P, Broschat SL. Aggressive region growing for speckle reduction in ultrasound images. Pattern Recog Lett 2003; 24:677-691.

Chinrungrueng C, Suvichakorn A. Fast edge-preserving noise reduction for ultrasound images. IEEE Trans Nucl Sci 2001;48:849-854.

Donoho DL. De-noising by soft-thresholding. IEEE Trans Inform Theory 1995;41:613-627.

Dutt V, Greenleaf JF. Statistics of the log-compressed echo envelope. J Accoust Soc Am 1996;99:3817-3825.

George N, Christensen CR, Bennerr JS, Guenther BD. Speckle noise in displays. J Opt Soc Am 1976;66:1282-1290.

Hao X, Gao S, Gao X. A novel multiscale nonlinear thresholding method for ultrasonic speckle suppressing. IEEE Trans Med Imaging 1999;18(9):787-794.

Haralick RM, Watson L. A facet model for image data. Comput Graph Image Process 1981;15:113-129.

Jain AK. Fundamentals of digital image processing. Englewood Cliffs, NJ: Prentice-Hall, 1989.

Jakeman E, Tough RJA. Generalized $K$ distribution: A statistical model for weak scattering. J Opt Soc Am 1987;4:1764-1772.

Karaman M, Alper Kutay M, Bozdagi G. An adaptive speckle suppression filter for medical ultrasound imaging. IEEE Trans Med Imaging 1995;14(2):283-292.

Kirsch RA. Computer determination of the constitute structure of biological images. Comput Biomed Res 1971;4:315-328.

Koivunen V. A robust nonlinear filter for image restoration. IEEE Trans Image Process 1995;4:569-578.

Kotropoulos C. Nonlinear ultrasonic image processing based on signaladaptive filters and self-organizing neural networks. IEEE Trans Image Process 1994;3(1):65-77.

Kozma A, Christensen CR. Effect of speckle on resolution. J Opt Soc Am 1976;66:1257-1260.
Li PC, O’Donnell M. Elevational spatial compounding. Ultrason Imaging 1994;16:176-189.

Loupas T, McDicken WN, Allan PL. An adaptive weighted median filter for speckle suppression in medical ultrasonic images. IEEE Trans Circ Sys 1989;36:129-135.

Macey KE, Page WH. Wavelet median denoising of ultrasound images. Proc SPIE 2002;4684(II):1151-1160.

Quistgaard JU. Signal acquisition and processing in medical diagnostic ultrasound. IEEE Signal Proc Mag 1997;1:67-74.

Rakotomamonjy A, Marche P. Wavelet-based enhancement of lesion detectability in ultrasound B-scan images. Proc IEEE Eng Med Biol Soc 1998;2:808-811.

Thijssen JM. Ultrasonic speckle formation, analysis and processing applied to tissue characterization. Patt Recog Lett 2003;24:659-675.

Thijssen JM, Oosterveld BJ. Texture in tissue echogram: Speckle or information? J Ultrasound Med 1990;9:215-229.

Toothaker LE. Multiple comparison procedures. Newbury Park, California: Sage Publications, 1993.

Tuthill TA, Sperry RH, Parker KJ. Deviation from Rayleigh statistics in ultrasonic speckle. Ultrason Imaging 1988;10:81-90.

Wanger RF, Smith SW, Sandrik JM, Lopez H. Statistics of speckle in ultrasound B-scans. IEEE Trans Sonics Ultrason 1983;SU-30:156163.

Yang PM, Huang GT, Lin JT, et al. Ultrasonography in the diagnosis of benign diffuse parenchymal liver diseases: A prospective study. J Formosan Med Assoc 1988;87:966-977.

Zong X, Laine AF, Geiser EA. Speckle reduction and contrast enhancement of echocardiograms via multiscale nonlinear processing. IEEE Trans Med Imaging 1998;17(4):532-540.

\section{APPENDIX 1}

\section{DERIVATION OF THE VARIANCE RELATION}

To derive the variance relation, by definition;

$$
\begin{gathered}
\sigma_{m}^{2}(x, y)=\gamma \sum_{\forall(i, j) \in W_{m}(x, y)}\left(s(i, j)+S^{1 / 2}(i, j)-\gamma \sum_{\forall(i, j) \in W_{m}(x, y)}(s(i, j)\right. \\
\left.\left.+s^{1 / 2}(i, j) n(i, j)\right)\right)^{2} \\
=\gamma \sum_{\forall(i, j) \in W_{m}(x, y)} X(i, j)+2 \gamma \sum_{\forall(i, j) \in W_{m}(x, y)} Y(i, j)+\gamma \sum_{\forall(i, j) \in W_{m}(x, y)} Z(i, j),
\end{gathered}
$$

where

$$
\begin{gathered}
X(i, j)=(s(i, j)-s(x, y))^{2} \\
Y(i, j)=\left(( s ( i , j ) - s ( x , y ) ) \left(s^{1 / 2}(i, j) n(i, j)\right.\right. \\
\left.\left.-\gamma \sum_{\forall(p, q) \in W_{m}(x, y)} s^{1 / 2}(p, q) n(p, q)\right)\right) \\
Z(i, j)=\left(s^{1 / 2}(i, j) n(i, j)-\gamma \sum_{\forall(p, q) \in W_{m}(x, y)} s^{1 / 2}(p, q) n(p, q)\right)^{2} .
\end{gathered}
$$

By showing that:

$$
\lim _{m \rightarrow \infty} E\left(\left(\gamma \sum_{\forall(i, j) \in W_{m}(x, y)} Y(i, j)-E(Y(x, y))\right)^{2}\right)=0 .
$$


and

$$
\lim _{m \rightarrow \infty} E\left(\left(\gamma \sum_{\forall(i, j) \in W_{m}(x, y)} Z(i, j)-E(Z(x, y))\right)^{2}\right)=0
$$

The second and the third terms of the second equality in eqn (25), $\gamma \Sigma \forall(i, j) \in W_{m}(x, y) Y(i, j)$ and $\gamma \Sigma \forall(i, j) \in W_{m}(i, j) Z(i, j)$, may be considered equal to $E(Y(x, y))$ and $E(Z(x, y))$ respectively, in the mean-squared sense for the sufficiently large window $W_{m}$ used in this study. Because $E(Y(x, y))=0$ and $E(Z(x, y))=(1-\gamma)^{2} s(x, y) \sigma_{n}^{2}$, eqn (25) may be reduced to:

$$
\sigma_{m}^{2}(x, y)=\gamma\left(\sum_{\forall(i, j) \in W_{m}(x, y)}(s(i, j)-s(x, y))^{2}\right)+(1-\gamma) s(x, y) \sigma_{n}^{2} .
$$

From eqn (31), the variance within the $m \times m$ window centered at each pixel $(x, y)$ is composed of two ingredients. One is the variance contributed by the intensity variation on a slope and the other is due to the noise variance weighted by the pixel intensity. Because the first term of eqn (31) is the same for all pixels on the same slope facet,

$$
\begin{gathered}
\sigma_{m}^{2}\left(x^{\prime}, y^{\prime}\right)=\gamma\left(\sum_{\forall(i, j) \in W_{m}\left(x^{\prime}, y^{\prime}\right)}\left(s(i, j)-s\left(x^{\prime}, y^{\prime}\right)\right)^{2}\right) \\
+(1-\gamma)^{2} s\left(x^{\prime}, y^{\prime}\right) \sigma_{n}^{2}=\gamma\left(\sum_{\forall(i, j) \in W_{m}\left(x^{\prime}, y^{\prime}\right)}\left(s(i, j)-s\left(x^{\prime}, y^{\prime}\right)\right)^{2}\right) \\
+(1-\gamma) c s(x, y) \sigma_{n}^{2}, \\
\approx \sigma_{m}^{2}(x, y)+(1-\gamma)(c-1) \mu_{m}(x, y) \sigma_{n}^{2},
\end{gathered}
$$

which gives the variance relation. Note that $s(x, y)$ is approximated by $\mu_{m}(x, y)$ from the second line to the third line of eqn (32), because $E(\mu(x, y))=s(x, y)$. 